\title{
A New Surgical Approach for Aortic Root Aneurysm and Small Aortic Annulus
}

\author{
Mehmet Kaplan, MD, Anil Karaagac, MD, Mehmet Inanc Yesilkaya, MD, Yusuf Kagan Pocan, MD, \\ Hakki Aydogan, MD \\ Department of Cardiovascular Surgery, University of Health Sciences, Siyami Ersek Thoracic and Cardiovascular Surgery Training \\ and Research Hospital, Istanbul, Turkey
}

\section{ABSTRACT}

Reconstruction of the aortic root in patients with aortic root aneurysm and small aortic annulus is an intricate surgical procedure. Here, we describe our technique performed in 2 patients, that involved aortic root enlargement with grafting in conjunction with annular implantation of the composite graft with a prosthetic mechanical valve, which allowed us to avoid incompatibility. This novel method offers an alternative combined surgical approach for patients with small aortic annulus and aortic root aneurysm that preserves the structure of the valved conduit at the level of the aortic annulus.

\section{INTRODUCTION}

A Bentall procedure is a specific type of surgery performed for the correction of aortic root aneurysms complicated by aortic valvular pathology. Small aortic annulus is an uncommon pathology in patients with aortic root aneurysm [Svensson 1992; Freitas-Ferraz 2019]. We performed aortic root enlargement with Dacron patch and Bentall procedure in 2 patients, owing to the presence of aortic root aneurysm and aortic valve pathology with small aortic annulus.

This new surgical approach allows the use of a larger-size valved conduit for a small aortic annulus with aortic root enlargement, while preserving the structure of the valved conduit.

\section{CASE REPORT}

The first case was a 65 -year-old female patient with aneurysm of the aortic root and ascending aorta, insufficiency and stenosis of the aortic valve, and coronary artery disease. The respective diameters of the ascending aorta and aortic root were 5.8 and $5 \mathrm{~cm}$ by computerized tomography (CT) scan. A Bentall procedure with coronary artery bypass grafting was planned. Preoperative echocardiography showed an aortic annulus diameter of $22 \mathrm{~mm}$ and ascending aorta diameter of $53 \mathrm{~mm}$. Body surface area was $1.78 \mathrm{~m}^{2}$.

Received fanuary 25, 2020; received in revised form February 17, 2020; accepted February 19, 2020.

Correspondence: Prof Mebmet Kaplan, MD, Prof Atatürk Mah, Meric Cad, Mercan 11. Blok, D: 38, 34758, Atasehir, Istanbul, Turkey; 90-532-270-3633 (e-mail:drmebmetkaplan@gmail.com).
The second patient was a 69-year old female who had severe aortic valvular insufficiency with an ascending aortic diameter of $7 \mathrm{~cm}$ and aortic root diameter of $5 \mathrm{~cm}$ by CT scan. Preoperative echocardiography showed an aortic annulus diameter of $22 \mathrm{~mm}$ and ascending aorta diameter of 70 $\mathrm{mm}$. Body surface area was $1.6 \mathrm{~m}^{2}$.

\section{Operative Technique}

Only median sternotomy incision was used for both patients. For cardiopulmonary bypass, arterial cannulation was performed at the aneurysmatic ascending aorta segment.

In the first patient, after performing distal anastomoses for coronary artery disease, the aortic valve was resected through an aortotomy. Because a $21-\mathrm{mm}$ valve size was found to be inappropriate for the aortic annulus, aortic root enlargement was performed with Dacron patch, and a \#23/26 valved conduit (S1274069-B, Vascutek, Sorin Group Italia, Saluggia, Italy) was implanted to avoid a mismatch between the patient and the prosthesis, as well as between the graft and the distal aorta.

In the second patient, a decision to perform aortic root enlargement was made based on measurements with a $21-\mathrm{mm}$ tester that showed a narrower aortic annulus. Because the graft size for a $23-\mathrm{mm}$ valved conduit was $24 \mathrm{~mm}$, and because this graft diameter did not match that of the distal
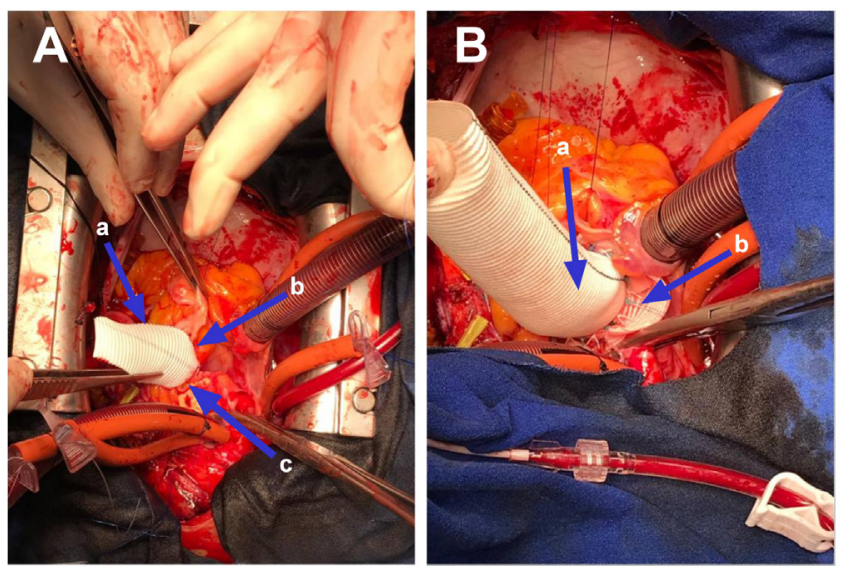

Figure 1. A, Aortic root enlargement with Dacron patch in the first patient. Arrows: a, aortic root enlargement graft; b, noncoronary aortic tissue; c, left coronary aortic tissue. B. The graft used for root enlargement and valved conduit, after implantation of the valved conduit in the first patient. Arrows: a, valved conduit; b, aortic root enlargement graft. 

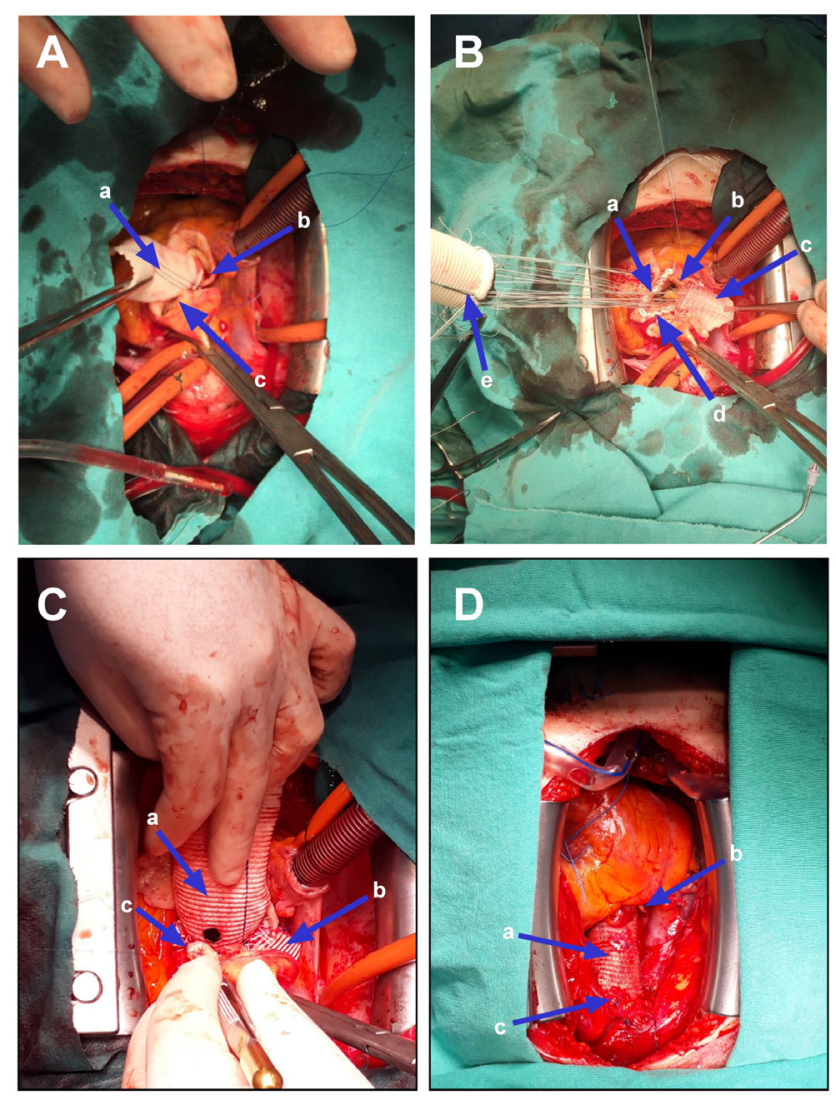

Figure 2. A, Aortic root enlargement with Dacron patch in the second patient. Arrows: a, aortic root enlargement graft; b, noncoronary aortic tissue; c, left coronary aortic tissue. B, Mitral valve sutures at the valved conduit and aortic annulus, together with the graft used for root enlargement, in the second patient. Arrows: a, right coronary annulus of aortic valve; b, noncoronary annulus; c, aortic root enlargement graft; $d$, left coronary annulus; e, valved conduit. C, Valved conduit placed onto the graft used for root enlargement and the left main button anastomosis in the second patient. Arrows: a, valved conduit; b, aortic root enlargement graft; $c$, left main coronary artery button. D, The final appearance after surgery in the second patient. Arrows: a, valved-conduit; b, aortic root enlargement graft; $c$, site for cannulation through aortic anastomosis.

aortic diameter, a 25/28-mm valved conduit (SJM, Masters Series Aortic Valved Graft, with Hemashield graft technology, St Jude Medical, Saint Paul, MN) was implanted after root enlargement.

In both patients, the aortotomy incision for aortic root enlargement was advanced through the commissure between the left and noncoronary cusps to subannular level toward aorticomitral continuity, up to the level of the left atrial roof. The Dacron patch was sutured onto the triangular space formed, starting from the lowest point of the incision (ie, technique of Nunez) [Nunez 1983] (Figures 1A and 2A). The valved conduit was then implanted to the newly formed aortic root using noncontinuous pledgeted mitral valve sutures (because the pledgets were large) (Figure 2B). After implantation, the Dacron patch used for aortic root enlargement was seen to extend outside the valved conduit (Figures $1 \mathrm{~B}$ and $2 \mathrm{C}$ ). The final appearance (valved-conduit, aortic root enlargement graft, and site for cannulation through aortic anastomosis after surgery in the second patient) is shown in Figure 2D.

After completion of the aortic root enlargement and valved conduit implantation, coronary button anastomoses were performed. The aneurysmatic aortic root and ascending aortic tissue were then resected up to $1 \mathrm{~cm}$ close to the brachiocephalic artery under deep hypothermic circulatory arrest (DHCA) at $18^{\circ} \mathrm{C}$. As a part of our routine procedure, open-distal anastomosis was performed between the graft and distal aortic tissue. During DHCA, no cerebral perfusion was performed [Kaplan 2015].

Before completion of the anastomosis between graft and the distal aortic tissue, the required suture safety precautions were observed, and the aortic cannula was placed on the top of the anastomosis (Kaplan technique [Kaplan 2019]). The circulation was reinitiated, with DHCA durations of 31 and 34 minutes for each patient. The routine procedure was completed uneventfully in both patients, the first case also having proximal saphenous vein anastomoses. After decannulation, no bleeding occurred at the aortic cannulation site at the anastomosis.

Postoperatively, patients had no hemodynamic or neurological complications and were extubated on the day of surgery, transferred to the wards on postoperative day 1, and discharged with full recovery at day 10 .

\section{Follow-Up}

Echocardiography showed functional mechanical valves, normal ejection fraction, and no significant valvular gradients at 8 and 2 months of follow up for the first and second patient, respectively. Both patients had Class I functional capacity, with no clinical complaints.

\section{DISCUSSION}

So far, few studies have addressed the management of aortic root aneurysm cases with small aortic annulus [Yakut 2001; Urbanski 2002]. Yakut recommended creation of a "flange" by eversion of the proximal end of the graft and suturing the graft using continuous Prolene sutures, after suture fixation of the mechanic valve within the graft at 4 points [Yakut 2001]. The flange is then reverted back to its original position, and the end of the graft is sutured to the aortic annulus. In contrast, our solution for patients in need of aortic root enlargement is at the level of the annulus, involving aortic root enlargement and preserving the structure of the valved conduit. This allows us to use larger-sized ascending aorta grafts, while preserving the structure of the valved conduit. In the technique of Yakut [2001], further coronary mobilization is required because of the upward displacement of the valve within the graft. In contrast, a button anastomosis just above the level of the aortic annulus is performed in our technique, negating the need for further coronary mobilization.

In such patients, Urbanski [2002] recommended suturing interiorly a mechanical valve larger than the size of the aortic 
annulus, 3 to $4 \mathrm{~mm}$ distant from the proximal end of a tube graft, together with implantation of the end of this graft onto the aortic annulus. In that case series, 20-, 21-, and 23-mm valves were used in 1,5 , and 4 patients, respectively, whereas 23/26and 25/28-mm valved conduits were used in our patients.

Both Yakut [2001] and Urbanski [2002] changed the configuration of the graft to achieve a match with the small aortic annulus, by displacing the mechanical valves to a point that was 3 to $4 \mathrm{~mm}$ distal to the proximal end of the graft, with suturing of the proximal end of the graft to the annulus. Conversely, we preferred to perform aortic root enlargement with Dacron patches in line with our routine approach, and then we combined this technique with the modified Bentall procedure. This allowed us to directly implant a larger-size valved conduit to the newly formed aortic annulus, replacing the aortic root and ascending aorta with an appropriately sized graft. Whereas the techniques described by Yakut [2001] and Urbanski [2002] did not involve a rigid ring at the level of the aortic annulus, we placed a rigid ring physically supporting the aortic annulus by placing the valved conduit at the aortic root. In considering techniques in which the mechanical valve is not at the tip of the graft but within the graft, a narrow area is created in the left ventricular outlet at the subvalvular annulus level, if root enlargement is not performed for a small aortic annulus.

The novel surgical approach described here for the aortic root and aortic valve surgery (ie, Bentall with aortic root enlargement) offers a new solution for pathologies involving the aortic root via the use of larger-size aortic valve and vascular graft, which preserves the structure of the valved conduit.

\section{REFERENCES}

Freitas-Ferraz AB, Tirado-Conte G, Dagenais F, et al. Aortic stenosis and small aortic annulus. Circulation 2019;139:2685-2702.

Kaplan M, Temur B, Can T, Abay G, Olsun A, Aydogan H. Open distal anastomosis technique for ascending aortic aneurysm repair without cerebral perfusion. Heart Surg Forum 2015;18(4):124-128.

Kaplan M, Karaagac A, Yesilkaya MI, Can T, Pocan YK, Aydogan H. a new arterial cannulation technique: Arterial cannulation through aortic anastomosis ("Kaplan" technique). Heart Surg Forum 2019;22(1):8-10.

Nunez L, Aguado MG, Pinto AG, Larrea JL. Enlargement of the aortic annulus by resecting the commissure between the left and noncoronary cusps. Tex Heart Inst J 1983;10:301-303.

Svensson LG, Crawford ES, Hess KR, Coselli JS, Safi HJ. Composite valve graft replacement of the proximal aorta: Comparison of techniques in 348 patients. Ann Thorac Surg 1992;54:427-437.

Urbanski PP. Complete aortic root replacement in patients with small aortic annulus. Ann Thorac Surg 2002;73:725-728.

Yakut C. A new modified Bentall procedure: The flanged technique. Ann Thorac Surg 2001;71:2050-2052. 